\title{
Late-Cutting Lipid-Soluble Alfalfa Extract Beneficially Modulates the Colon Microbiota to Protect Mouse Body Weight during Citrobacter Rodentium Challenge
}

\section{DOI:10.31274/air.11911}

Krysten Fries-Craft, Graduate Research Assistant; Justin M. Anast, Graduate Research Assistant; Stephan Schmitz-Esser, Associate Professor Elizabeth Bobeck, Assistant Professor; Department of Animal Science, Iowa State University

\section{Summary and Implications}

Mouse models allow for detailed preliminary research into the health-promoting effects of novel feed additives to identify candidates for livestock application. Alfalfa is a potential source of health-promoting feed additives, but detailed findings on factors that maximize its benefits are lacking. The study objective was to evaluate the effects of feeding late and early alfalfa as hay, aqueous, and lipidsoluble extracts on the body weight (BW) and colon microbiota of mice before and after pathogen challenge. A total of 98 female 6-week-old C57BL/6J mice were weighed and assigned to 1 of 7 diets consisting of basal diet \pm hay (9\%), aqueous extract $(0.25 \%)$, and chloroform extract $(0.25 \%)$ of late and early cutting alfalfa. After $14 \mathrm{~d}, 6$ mice/treatment were euthanized for baseline colon digesta sampling. The remaining animals were orally inoculated with Citrobacter rodentium and 4 mice/ treatment were euthanized for colon digesta collection at 4 and $21 \mathrm{~d}$ postinoculation (pi). While observed patterns in BW were not statistically significant, numerical improvements in early and late days post-inoculation (pi) indicated a protective effect of lipid-soluble extracts on BW during bacterial challenge. Corresponding to these observations, late cutting chloroform extracts significantly reduced pathogen abundance in the colon at 4dpi while increasing the abundance of beneficial community members at $21 \mathrm{dpi}(P=$ $0.02)$. These findings in mice support future research into the effects of late-cutting chloroform alfalfa extracts in livestock diets.

\section{Introduction}

Plant-based feed ingredients offer a versatile way to incorporate potentially beneficial compounds into animal diets; however, variability in chemical profiles due to environmental factors make it difficult to identify ways to maximize conferred benefits. Alfalfa, a forage crop already used in livestock nutrition, is known to improve animal health. Specific plant components responsible for these outcomes have not been investigated in detail. These health benefits may be associated with altered intestinal microbial communities that have indirect impacts on health through the production of secondary metabolites but may show differential response to dietary alfalfa fed whole or as an extract. The intestinal microbiota of livestock is not as welldefined as other available animal models and complicates detailed investigation into the effects of feeding alfalfa. This study used a well-defined mouse model (C57BL/6J) and a rodent-specific gastrointestinal pathogen (Citrobacter rodentium) to identify the effects of feeding early and latecutting alfalfa on the BW and intestinal microbiota of mice.

\section{Materials and Methods}

Early $\left(1^{\text {st }}\right)$ and late $\left(5^{\text {th }}\right)$ cutting alfalfa from the same field was ground and extracted into lipid- or water-soluble fractions prior to incorporation into animal diets. Female 6week-old C57BL/6J mice (98 total; Jackson Laboratories, Bar Harbor, ME) were given a $7 \mathrm{~d}$ acclimation period to allow the microbiota to stabilize after transport and transition to a commercially available diet (Teklad Global $18 \%$ protein rodent diet, Envigo Teklad, Madison,WI). At $\mathrm{d} 0$, mice were weighed and assigned to 1 of 7 treatments consisting of the commercially available basal diet (control) $\pm 9 \%$ hay, $0.25 \%$ aqueous extract, or $0.25 \%$ chloroform extract from $1^{\text {st }}$ or $5^{\text {th }}$ cutting alfalfa.

At d14, all mice were weighed and 6 mice/ treatment were euthanized for baseline colon digesta collection. The remaining mice were orally inoculated with $200 \mu$ of $2 \times 10^{10}$ colony forming units (CFU) of $C$. rodentium. Following inoculation, mice were weighed daily and colon digesta samples were collected from 4 mice/ treatment at $4 \mathrm{~d}$ and 21 dpi. DNA was extracted from collected digesta using a commercially available kit and 16S rRNA gene amplicon sequencing was performed by the DNA Facility at Iowa State University.

Body weight data were analyzed using the MIXED procedure (SAS 9.4) with the effects of alfalfa form and cutting nested within the control to account for the $2 * 3+1$ factorial treatment design with 2 alfalfa cuttings, 3 supplementation forms, and the control. Obtained DNA sequences were quality screened using Mothur software (v.1.40.4) and clustered into operational taxonomic units (OTUs) at $97 \%$ similarity corresponding to genus. OTU-

Copyright $(\mathcal{C} 2020$ by the Authors. This is an open access article published under the CC BY-NC license (https://creativecommons.org/licenses/by-nc/4.0/), which allows for non-commercial reuse with proper attribution. 
level comparisons were done using the LEfSe test in the Mothur software. Significance was denoted at $P \leq 0.05$ for all analyses.

\section{Results and Discussion}

Prior to inoculation, mouse BW was not affected by alfalfa supplementation, and uniformly decreased in the timepoints following $C$. rodentium inoculation across treatments. Throughout the post-inoculation period, BW showed numerically, but not statistically, different responses to different forms and cuttings (Figure 1). Most notably, mice fed $5^{\text {th }}$ cutting lipid-soluble extract returned to preinoculation BW 2d earlier than control. Only mice fed lipidsoluble alfalfa extracts had numerically greater BW compared to the control at later timepoints (Figure 1E,F). As a result, microbial community comparisons in control vs. lipid-soluble extract diets are presented as these were the only diets to contribute to an observable phenotypic change.

The most notable outcome observed was the 18.1-fold reduction of $C$. rodentium relative abundance in the colon digesta of mice fed $5^{\text {th }}$ cutting chloroform extract compared to the control $(P=0.02)$. At later timepoints, $5^{\text {th }}$ cutting lipid-soluble extracts increased the relative abundance of Akkermansia approximately 7-fold compared to the control $(P=0.02)$. Members of the Akkermansia genus have been associated with anti-inflammatory effects and are generally thought to be beneficial.

Overall, these results suggest that lipid-soluble latecutting alfalfa components modulated the intestinal microbiota to reduce pathogen abundance in early stages of C. rodentium infection and increase beneficial community members during inoculation resolution. These observations were specific to late-cutting alfalfa and correspond to improved BW at early and later post-inoculation timepoints. Feed additives that enable livestock to clear microbial challenges more quickly than the animal would without supplementation could provide invaluable benefit to industry. These outcomes support further investigation into the effect of lipid-soluble late-cutting alfalfa extracts on livestock health. 
A.

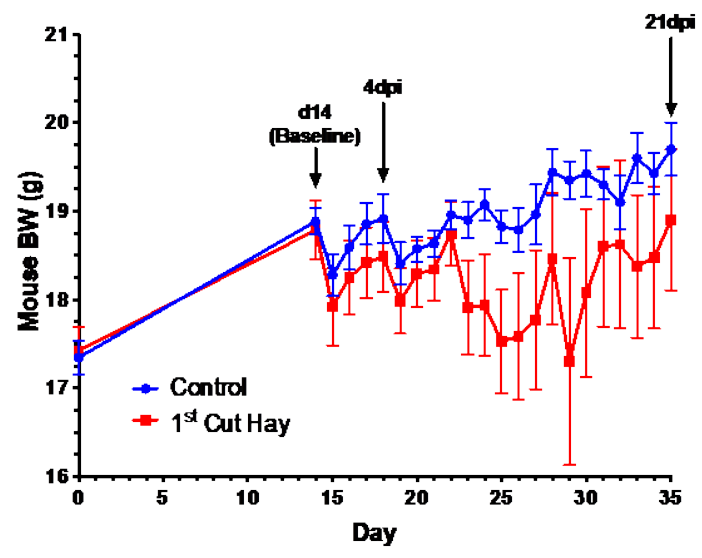

C.

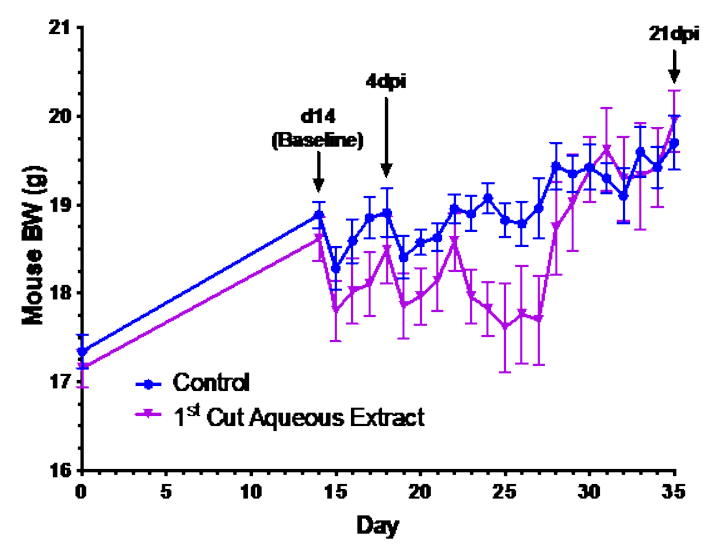

E.

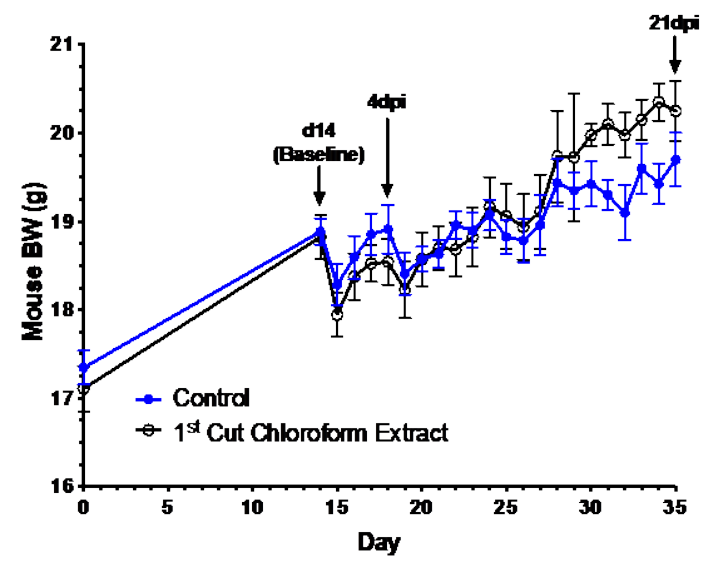

B.

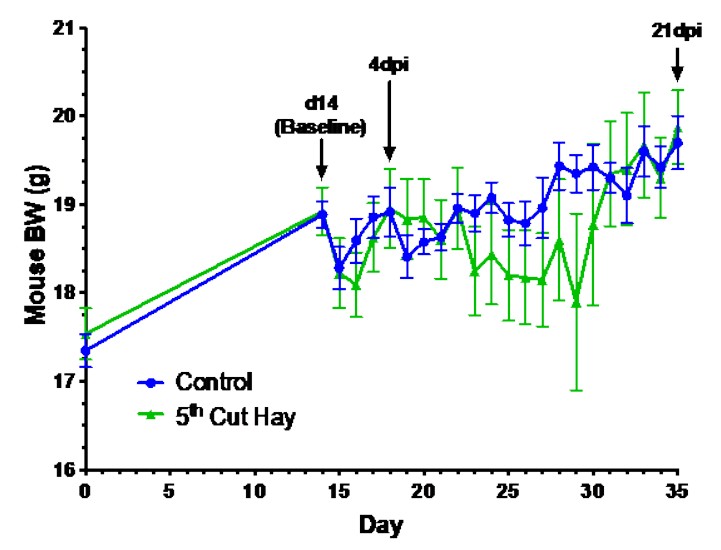

D.

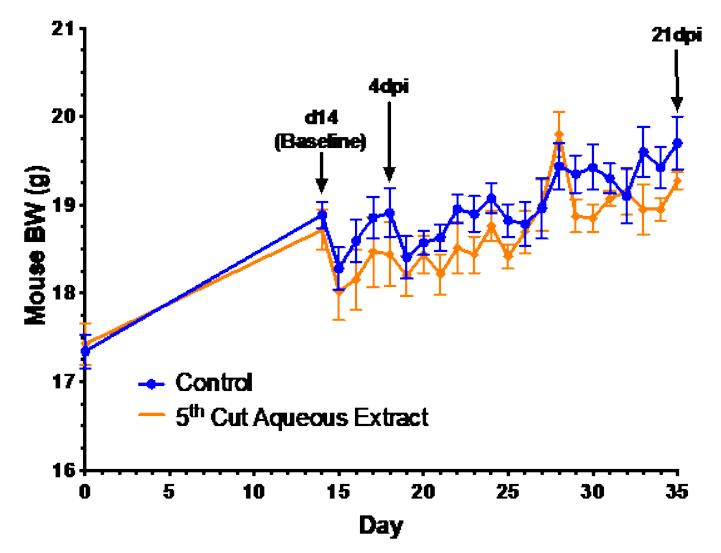

F.

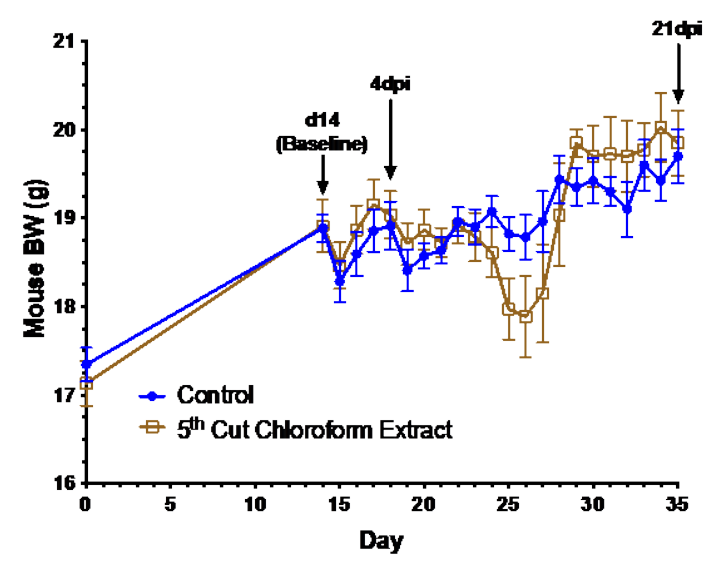

Figure 1: Body weight comparison of 6-week-old female C57BL/6J mice fed diets containing (A) $1^{\text {st }}$ cut alfalfa hay, (B) $5^{\text {th }}$ cut alfalfa hay, (C) aqueous extract of $1^{\text {st }}$ cut alfalfa, (D) aqueous extract of $5^{\text {th }}$ cut alfalfa, (E) chloroform extract of $1^{\text {st }}$ cut alfalfa, or (F) chloroform extract of $5^{\text {th }}$ cut alfalfa versus a control diet without supplemental alfalfa (blue line). Mice were orally inoculated at d14 (baseline) with Citrobacter rodentium and the main timepoints for microbiota analysis are denoted in each panel at 4 and $21 \mathrm{~d}$ post-inoculation. 